\title{
Possible Predictive Factors for In-hospital Cardiac Arrest in Patients with Cancer: A Retrospective Single Center Study
}

\author{
Muhammad Sardar $^{1}$, Nasreen Shaikh ${ }^{2}$, Saad Ullah Malik ${ }^{3}$, Faiz Anwer ${ }^{4}$, Patrick Lee ${ }^{5}$, David Sharon ${ }^{5}$, \\ Margaret HH Eng ${ }^{1}$ \\ 1. Internal Medicine, Monmouth Medical Center, Long Branch, USA 2. Department of Internal Medicine, Monmouth \\ Medical Center, Long Branch, USA 3. Hematology Oncology, University of Arizona, Tuscon, USA 4. Hematology and \\ Oncology, University of Arizona, Tucson, USA 5. Hematology Oncology, Monmouth Medical Center, Long Branch, USA
}

Corresponding author: Muhammad Sardar, muhammad.sardar527@gmail.com

\begin{abstract}
Background: Despite cancer being the second most common cause of death in the United States, more people are living longer after the diagnosis of cancer than before. Healthcare workers will be treating an increasing number of patients with cancer. Various studies have identified predictors of cardiac arrest in the general population, however, none have been done to identify such factors in cancer patients who form a more vulnerable group with lower survival rate following cardiac arrest.
\end{abstract}

Methods: We retrospectively analysed charts of all patients with active cancer who experienced in-hospital cardiac arrest (IHCA) and underwent cardio-pulmonary resuscitation (CPR) from January 2015 to December 2017 at our hospital ( $\mathrm{n}=44$, group A). We compared this group to 44 consecutive patients with active cancer admitted to the oncology unit who did not experience cardiac arrest ( $n=44$, group B). We excluded patients in remission.

Results: Both the groups were comparable in terms of age $(69 \pm 14$ vs $68 \pm 15, \mathrm{p}=0.776)$ and gender distribution ( $50 \%$ vs $56 \%$ males, $\mathrm{p}=0.521)$. Prevalence of coronary artery disease (CAD) $(25 \%$ vs $11 \%$, $\mathrm{p}=0.097$ ), hypertension ( $68 \%$ vs $66 \%, \mathrm{p}=0.821$ ), hyperlipidaemia ( $34 \%$ in both groups, $\mathrm{p}=1.000$ ), tobacco abuse ( $18 \%$ vs $27 \%$, $\mathrm{p}=0.308$ ), and diabetes mellitus ( $34 \%$ vs $23 \%$, $\mathrm{p}=0.237$ ) was not significantly different between the two groups. Group with cardiac arrest had significantly higher alanine aminotransferase $(100 \mathrm{U} / \mathrm{L} \pm 150$ vs $47 \mathrm{U} / \mathrm{L} \pm 87, \mathrm{p}=0.043)$, alkaline phosphatase ( $288 \mathrm{U} / \mathrm{L} \pm 512$ vs $118 \mathrm{U} / \mathrm{L} \pm 80, \mathrm{p}=0.032)$, creatinine $(1.8 \mathrm{mg} / \mathrm{dl}$ $\pm 1.74 \mathrm{vs} 1.1 \mathrm{mg} / \mathrm{dl} \pm 0.76, \mathrm{p}=0.023)$, international normalised ratio (INR) $(2.1 \pm 1.5 \mathrm{vs} 1.2 \pm 0.5, \mathrm{p}=0.005)$, and lower estimated -glomerular filtration rate $\left(43 \mathrm{~mL} / \mathrm{min} / 1.73 \mathrm{~m}^{2} \pm 17\right.$ vs $51 \mathrm{~mL} / \mathrm{min} / 1.73 \mathrm{~m}^{2} \pm 15$, $\left.\mathrm{p}=0.022\right)$ on admission. Group A also had significantly higher incidence of sepsis during the hospital course as compared to group B ( $30 \%$ vs $2 \%, \mathrm{p}<0.001$ ). In group A, $11.4 \%$ survived to discharge as compared to $95.5 \%$ in group B. Significantly higher number of patients in group B were taking chemotherapy $(77.27 \%$ vs $34.09 \%$, $\mathrm{p}=0.000046)$ and radiation therapy ( $65.9 \%$ vs $22.72 \%, \mathrm{p}=0.000046)$ as compared to group A.

Received 06/01/2018

Review began 06/09/2018 Review ended 06/13/2018 Published 06/18/2018

๑) Copyright 2018

Sardar et al. This is an open access article distributed under the terms of the Creative Commons Attribution License CC-BY 3.0., which permits unrestricted use, distribution, and reproduction in any medium, provided the original author and source are credited.
Conclusion: Cancer patients who experienced IHCA had worse renal and hepatic function; they were frequently diagnosed with sepsis and had similar cardiovascular risk factors as compared to cancer patients who did not experience cardiac arrest. Furthermore, a higher number of patients with active cancer who did not experience cardiac arrest were on chemotherapy, immunotherapy or radiation therapy.

Categories: Cardiology, Internal Medicine, Oncology

Keywords: cardiac arrest, predictors, cancer

\section{Introduction}

According to the Centers for Disease Control and Prevention (CDC), cancer was the second leading cause of death in 2015 with 595,930 deaths preceded only by heart disease as the number one cause of death [1]. In spite of cancer being the second leading cause of death, people diagnosed with cancer in the United States are living longer than before [2]. Healthcare providers will be expected to treat an increasing number of cancer patients in their practice; hence they should be able to identify patients with high risk of mortality.

Several scoring systems have been developed to identify patients on regular medical floors at high risk for adverse events. National Early Warning Score (NEWS), developed in 2012 by the Royal College of Physicians, is one such risk stratification system that takes into account vital signs (heart rate, respiratory rate, temperature, systolic blood pressure, oxygen saturation, and oxygen supplementation) and mental status to identify patients at risk of deterioration [3]. Electronic-Cardiac Arrest Risk Triage (e-CART) is a scoring system that incorporates ward vital signs as well as laboratory results and demographics for combined prediction of cardiac arrest, intensive care unit (ICU) transfer, and death [4]. Multiple studies have validated their use in real-time [5]. These scoring systems predict the probability of an adverse event in the general 
Patients with cancer are a more vulnerable group compared to the general patient population. Survival rates after in-hospital cardiac arrest (IHCA) in patients with advanced cancer are lower than the rest of the population. In a study by Bruckle et al., 7.4\% patients with advanced cancer who had IHCA survived to discharge as compared to $13.4 \%$ without advanced cancer [6]. A knowledge of predictors of cardiac arrest in this population would help physicians act in a timely manner to avert this adverse event.

No study has been done to identify factors predicting cardiac arrest in cancer patients. We aim to compare demographics, vital signs, laboratory results, electrocardiogram (EKG) characteristics, and cancer characteristics of patients with active cancer who suffered IHCA to patients with active cancer who did not experience IHCA at our hospital.

\section{Materials And Methods}

Patients who experienced cardiac arrest and underwent cardio-pulmonary resuscitation (CPR) at Monmouth Medical Center from January 2015 to December 2017 were identified. After thorough chart analysis, those patients who had active cancer were included in the study. Active cancer was defined as patients having a documented objective evidence of cancer and who were not in remission. We excluded patients on hospice care, patients who experienced cardiac arrest in the field, and those with the order of do not resuscitate. These patients formed group A $(n=44)$. Group B consisted of 44 consecutive patients with active cancer admitted to the oncology unit who did not experience cardiac arrest from November 2017 to December 2017.

Baseline demographic and clinical characteristics, type and stage of cancer, medications, vital signs, complete blood count and comprehensive metabolic panel on the day of admission, and presence or absence of sepsis during hospital course were compared between the two groups. 12-lead EKG on admission was also compared. Surgical resection of the primary tumor, treatment with chemotherapy or radiation therapy was considered positive if it was within six months of admission.

Categorical variables were expressed as numbers with percentages and continuous variables were expressed as mean \pm standard deviation. Continuous variables were compared between the two groups using Student's t-test or Mann-Whitney U test. Categorical variables were compared using chi-squared test or Fischer's exact test. Statistical significance was defined as a p value $<0.05$. All data were analysed using SPSS 20.0 (SPSS Inc Chicago, Illinois, USA).

\section{Results}

Among 209 patients with IHCA followed by cardiopulmonary resuscitation from January 2015 to December 2017,44 patients had active cancer $(21.05 \%)$. The mean age of group with cardiac arrest (group A) was $69.14 \pm 13.91$ compared to $68.25 \pm 15.24$ in the group with the absence of cardiac arrest (group B), the difference was not significant. There was no significant difference in the prevalence of coronary artery disease (CAD), hyperlipidaemia, hypertension, diabetes mellitus, tobacco abuse, chronic obstructive pulmonary disease (COPD), and congestive heart failure (CHF) between the two groups. Significantly higher number of patients developed sepsis during hospital course in the group with cardiac arrest (29.54\% vs $2.27 \%, \mathrm{p}=0.00047)$. Table 1 summarizes the baseline demographic and clinical characteristics. 


\section{Cureus}

\begin{tabular}{|c|c|c|c|}
\hline Variable & Cardiac arrest $(n=44)$ & No cardiac arrest $(n=44)$ & $P$ value \\
\hline Age & $69.14 \pm 13.91$ & $68.25 \pm 15.24$ & 0.776 \\
\hline Male sex & $22(50 \%)$ & $25(56.8 \%)$ & 0.521 \\
\hline Coronary artery disease & $11(25 \%)$ & $5(11.4 \%)$ & 0.097 \\
\hline Hyperlipidemia & $15(34.1 \%)$ & $15(34.1 \%)$ & 1.000 \\
\hline Hypertension & $30(68.2 \%)$ & $29(65.9 \%)$ & 0.821 \\
\hline Diabetes mellitus & $15(34.1 \%)$ & $10(22.7 \%)$ & 0.237 \\
\hline COPD & $4(9.1 \%)$ & $5(11.5 \%)$ & 0.725 \\
\hline Peripheral vascular disease & $5(11.4 \%)$ & $1(2.3 \%)$ & 0.091 \\
\hline Pulmonary embolism & $5(11.4 \%)$ & $1(2.3 \%)$ & 0.091 \\
\hline CHF & $8(18.2 \%)$ & $4(9.1 \%)$ & 0.214 \\
\hline Sepsis & $13(29.5 \%)$ & $1(2.3 \%)$ & 0.0004 \\
\hline Tobacco abuse & $8(18.2 \%)$ & $1(2.3 \%)$ & 0.308 \\
\hline
\end{tabular}

\section{TABLE 1: Patient characteristics}

COPD: chronic obstructive pulmonary disease; CHF: congestive heart failure.

\section{Cancer characteristics and treatment}

Both groups had 25\% (11/44) patients with haematological malignancy and 75\% (33/44) patients with solid tumours. Metastatic disease was seen in $29.5 \%$ of patients who had cardiac arrest compared to $47.8 \%$ of the patients who did not have cardiac arrest, however, results were not statistically significant. $77.3 \%$ of patients who did not have cardiac arrest were on active treatment (chemotherapy and/or immunotherapy) in the six months preceding admission compared to $34.1 \%$ who experienced cardiac arrest, the difference was statistically significant, $\mathrm{p}=0.000046$. Similarly, $15.9 \%$ in group A had radiation therapy in the six months preceding admission as compared to $65.9 \%$ in group $\mathrm{B}$, the difference was significant, $\mathrm{p}=0.00002$. Results are summarized in Table 2 .

\begin{tabular}{|c|c|c|c|}
\hline Variables & Cardiac arrest $(n=44)$ & No cardiac arrest $(n=44)$ & P Value \\
\hline Hematological malignancy & $11(25 \%)$ & $11(25 \%)$ & 1.000 \\
\hline Solid malignancy & $33(75 \%)$ & $33(75 \%)$ & 1.000 \\
\hline Metastasis & $13(29.5 \%)$ & $21(47.8 \%)$ & 0.067 \\
\hline Chemotherapy \pm immunotherapy & 15 (34.1\%) & $34(77.3 \%)$ & 0.000046 \\
\hline Radiotherapy & $7(15.9 \%)$ & $29(65.9 \%)$ & 0.000002 \\
\hline Surgery & $8(18.2 \%)$ & $9(20.5 \%)$ & 0.787 \\
\hline
\end{tabular}

TABLE 2: Cancer characteristics and treatment

\section{Vital signs and EKG on admission}

Blood pressure, heart rate, and temperature were not significantly different between the two groups. The group with cardiac arrest had significantly higher respiratory rates $(24.5 \pm 15.4 \mathrm{vs} 19.4 \pm 3.1, \mathrm{p}=0.034)$ and lower oxygen saturation $(95.4 \pm 3.6$ vs $97 \pm 2.5$, $\mathrm{p}=0.014$ ) however oxygen supplementation was similar in both groups. Patients who did not have cardiac arrest were more often in normal sinus rhythm on admission based on 12 -lead EKG $(1.8 \%$ in group A vs $50 \%$ in group B, $p$ value $=0.000085)$. The results are summarized in Table 3 and Table 4 . 


\section{Cureus}

\begin{tabular}{|c|c|c|c|}
\hline Variable & Cardiac arrest $(n=44)$ & No cardiac arrest $(n=44)$ & P Value \\
\hline Systolic blood pressure (mmHg) & $116.6 \pm 26.2$ & $125.5 \pm 26$ & 0.116 \\
\hline Diastolic blood pressure $(\mathrm{mmHg})$ & $67.1 \pm 18.2$ & $68.8 \pm 15.2$ & 0.631 \\
\hline Heart rate & $97.1 \pm 29.2$ & $92.5 \pm 21.7$ & 0.411 \\
\hline Respiratory rate & $24.5 \pm 15.4$ & $19.4 \pm 3.1$ & 0.034 \\
\hline Oxygen saturation & $95.4 \pm 3.6$ & $97 \pm 2.5$ & 0.014 \\
\hline Temperature & $97.9 \pm 1.4$ & 5 & 0.189 \\
\hline
\end{tabular}

\section{TABLE 3: Vital signs}

\begin{tabular}{|c|c|c|c|}
\hline Variable & Cardiac arrest $(n=44)$ & No cardiac arrest $(n=44)$ & $P$ value \\
\hline Normal Sinus rhythm & $5(1.8 \%)$ & $22(50 \%)$ & 0.000085 \\
\hline Sinus tachycardia & $15(34.1 \%)$ & $13(29.5 \%)$ & 0.209 \\
\hline Sinus bradycardia & $2(4.5 \%)$ & $1(2.3 \%)$ & 0.557 \\
\hline Heart block ( $1^{\text {st }} / 2^{\text {nd }} / 3^{\text {rd }}$ degree) & $2(4.5 \%)$ & 0 & 1.000 \\
\hline Bundle branch block (right or left) & $7(15.9 \%)$ & $2(4.5 \%)$ & 1.791 \\
\hline Atrial arrhythmia & $10(22.7 \%)$ & $3(6.8 \%)$ & 1.222 \\
\hline Ventricular arrhythmia & $4(9.1 \%)$ & $6(13.6 \%)$ & 0.715 \\
\hline Non-specific ST/T wave changes & $6(13.6 \%)$ & $2(4.5 \%)$ & 1.380 \\
\hline Paced rhythm & $1(2.3 \%)$ & $2(4.5 \%)$ & 0.557 \\
\hline
\end{tabular}

TABLE 4: Electrocardiogram (EKG) characteristics

$\mathrm{n}$ is higher than $\mathbf{4 4}$ in each group as some patients may have more than one finding on their EKG.

\section{Laboratory tests}

There was no significant difference in white blood cell count, platelet count and hemoglobin between the two groups on admission. International normalised ratio (INR) was significantly higher in the group with cardiac arrest $(2.1 \pm 1.6$ vs $1.3 \pm 0.5, \mathrm{p}=0.005)$. Sodium, potassium and bicarbonate levels were not significantly different between the two groups. In the group with cardiac arrest, magnesium ( $2.2 \mathrm{vs} 1.4$, $\mathrm{p}=0.002$ ) and phosphorous ( 4.1 vs $3.1, \mathrm{p}=0.041)$ were significantly higher. Alanine aminotransferase $(100.4 \pm$ 149.9 vs $46.8 \pm 86.8, \mathrm{p}=0.043)$ and alkaline phosphatase $(288 \pm 512.8$ vs $118.3 \pm 80.2,0.032)$ were significantly higher in group A. Aspartate aminotransferase was not significantly different. Patients with cardiac arrest also had significantly higher serum creatinine on admission $(1.8 \pm 1.7$ vs $1.2 \pm 0.8 \mathrm{p}=0.023)$, lower estimated glomerular filtration rate $(44 \pm 16.8$ vs $51.8 \pm 14.6, \mathrm{p}=0.022)$ and higher blood urea nitrogen $(42.6 \pm 29.8 \mathrm{vs}$ $26.3 \pm 17.5, \mathrm{p}=0.002)$. Results are summarized in Table 5 . 


\section{Cureus}

\begin{tabular}{|c|c|c|c|}
\hline Variable & Cardiac arrest (n=44) & No cardiac arrest $(n=44)$ & P Value \\
\hline WBC & $29.6 \pm 106.2$ & $14.0 \pm 26$ & 0.351 \\
\hline Hemoglobin & $10.9 \pm 2.6$ & $10.3 \pm 2.7$ & 0.800 \\
\hline Platelets & $186 \pm 127.0$ & $225.9 \pm 138.9$ & 0.162 \\
\hline Sodium & $137.7 \pm 5.1$ & $137.6136 \pm 6.2$ & 0.926 \\
\hline Potassium & $4.4 \pm 0.8$ & $4.4 \pm 0.6$ & 0.988 \\
\hline Bicarbonate & $26.3 \pm 7.5$ & $27.4 \pm 4.6$ & 0.378 \\
\hline Magnesium & $2.2 \pm 0.49$ & $1.4 \pm 0.4$ & 0.002 \\
\hline Phosphorus & $4.1 \pm 2.2$ & $3.1 \pm 0.9$ & 0.041 \\
\hline INR & $2.1 \pm 1.6$ & $1.3 \pm 0.5$ & 0.005 \\
\hline AST & $177.4 \pm 375.3$ & $75.5 \pm 169.0$ & 0.104 \\
\hline ALT & $100.4 \pm 149.9$ & $46.8 \pm 86.8$ & 0.043 \\
\hline Alkaline phosphatase & $288 \pm 512.8$ & $118.3 \pm 80.2$ & 0.032 \\
\hline Creatinine & $1.8 \pm 1.7$ & $1.2 \pm 0.8$ & 0.023 \\
\hline eGFR & $44 \pm 16.8$ & $51.8 \pm 14.6$ & 0.022 \\
\hline BUN & $42.6 \pm 29.8$ & $26.3 \pm 17.5$ & 0.002 \\
\hline Troponins & $0.73 \pm 1.9$ & $0.14 \pm 0.48$ & 0.238 \\
\hline BNP & $821.6 \pm 594.0$ & $88 \pm 46.2$ & 0.060 \\
\hline
\end{tabular}

\section{TABLE 5: Laboratory data}

WBC: white blood cell count; INR: international normalised ratio; AST: aspartate transaminase; ALT: alanine transaminase; eGFR: estimated glomerular filtration rate; BUN: blood urea nitrogen; BNP: brain natriuretic peptide.

\section{Medications}

There was no significant difference in the medications that the patients were taking on admission namely antiplatelet, anticoagulants, beta blockers, calcium channel blockers (dihydropyridines \& nondihydropyridines), angiotensin-converting enzyme inhibitors/angiotensin receptor blockers, and statins. Table 6 shows the medications in both groups.

\begin{tabular}{|c|c|c|c|}
\hline Variable & Cardiac arrest $(n=44)$ & No cardiac arrest $(n=44)$ & P Value \\
\hline Antiplatelets & $13(29.54 \%)$ & $9(20.45 \%)$ & 0.325 \\
\hline Anticoagulants & $10(22.27 \%)$ & $8(18.18 \%)$ & 0.597 \\
\hline Beta blockers & $23(52.27 \%)$ & $15(34.09 \%)$ & 0.085 \\
\hline Calcium channel blockers & $8(18.18 \%)$ & $11(25 \%)$ & 0.437 \\
\hline Statins & $13(29.54 \%)$ & $12(27.27 \%)$ & 0.813 \\
\hline ACE-I/ARBS & $13(29.54 \%)$ & $11(25 \%)$ & 0.632 \\
\hline
\end{tabular}

\section{TABLE 6: Medications}

ACE-I/ARBs: angiotensin converting enzyme inhibitor/angiotensin receptor blockers.

\section{Characteristics of cardiac arrest}


$50 \%$ of the cardiac arrest rhythms were pulseless electrical activity, $31.81 \%$ were asystole and $15.9 \%$ were ventricular fibrillation or pulseless ventricular tachycardia. $11.36 \%$ of the patients with cardiac arrest survived to discharge. However, one patient died within three months after discharge. In comparison, 95.5\% patients in the group B survived to discharge.

\section{Discussion}

Our study aimed to identify factors that could predict the occurrence of IHCA in patients with active cancer so that pre-emptive interventions can be made to avert this adverse event. We found that evaluation of vital signs, laboratory results, and EKG findings can help identify patients at risk of developing IHCA.

Cancer patients who experienced IHCA were found to have worse hepatic and renal function on admission as compared to those who did not have IHCA. Various studies have shown that the presence of chronic kidney disease is an independent risk factor for sudden cardiac death even in the absence of CAD, CHF, and structural heart disease [7]. Chronic renal insufficiency leads to cardiac fibrosis and coronary artery calcification [8-14]. Cardiac structural remodeling in addition to renal disease associated with electrical abnormalities predispose patients to cardiac arrhythmias increasing the risk of sudden cardiac arrest [15]. Renal insufficiency is not only predictive of sudden cardiac death but the presence of acute kidney injury (AKI) in the post-cardiac arrest period has been associated with higher mortality [16]. Cancer patients generally have a higher incidence of AKI as many chemotherapeutic agents are nephrotoxic and these patients are also predisposed to pre-renal azotemia secondary to vomiting and diarrhea induced dehydration [17]. Samuels et al. found that even a small rise in creatinine has been associated with higher morbidity and mortality in critically ill cancer patients [18]. Hepatic dysfunction has also been associated with both systolic and diastolic dysfunction and electrophysiological abnormalities of the heart [19]. We saw that cancer patients who experienced IHCA had higher alanine aminotransferase, alkaline phosphatase, and INR on admission. These parameters have been included in several scoring systems that predict cardiac arrest in general floor patients [5]. Renal and hepatic function tests are available for most patients on admission and these derangements should be given consideration when triaging patients.

Our study also found that patients who did not experience cardiac arrest were more often found to be in normal sinus rhythm based on a 12-lead EKG on admission. Currently, there are no risk stratification calculators based on EKG findings even for the general population; however, high resting heart rate, nonsustained ventricular tachycardia, and heart rate turbulence may be helpful to identify patients at high risk for cardiac arrest when used in combination with other factors [20].

Sepsis was seen more commonly in patients with active cancer. The pathophysiological abnormalities associated with sepsis-like hypovolemia, hypoxemia, and acidosis not only confer a higher risk of cardiac arrest but also are poor predictors of survival after cardiac arrest [21]. Sepsis is known to have higher mortality in patients with cancer than those without cancer [22].

The type of cancer, hematological or solid organ, and the presence of metastatic disease were not predictive of IHCA in our study. Instead, we found that a significantly higher number of patients with cancer who suffered IHCA were not on any treatment for cancer. Most of these patients were newly diagnosed with cancer or had a recently detected recurrence and were scheduled to start treatment. In a study by Bleicher et al., it was found that in patients with breast cancer, a greater time to surgery was associated with lower overall and disease-specific survival [23]. Time from diagnosis to treatment is a vulnerable period and all efforts should be made to shorten it.

CAD is the most common cause of sudden cardiac death [24]. Interestingly, there was no statistically significant difference in the cardiovascular risk factors between the cancer patients who had cardiac arrest compared to patients who did not. Although CAD can cause cardiac arrest through mechanisms other than a myocardial infarction, none of our patients had cardiac arrest as a direct consequence of myocardial infarction [24-25]. Cardiovascular risk factors may have little predictive value for IHCA in cancer patients.

We acknowledge the limitations of our study. Our study was retrospective in nature so there is a possibility of inaccurate reporting of events. Moreoever, it was a single center study with a relatively small sample size. Although multiple studies have looked at the predictors of outcomes after IHCA, this was a first attempt to identify the predictors of having an IHCA in cancer patients. To validate the results of our study, we propose a future multicenter prospective trial where patients can be stratified into high risk versus low-risk category based on the factors identified by our study and then followed to compare the incidence of IHCA.

\section{Conclusions}

Hepatic and renal impairment on admission and the presence of sepsis are predictive of IHCA in patients with active cancer. On the other hand, type of cancer and the presence of metastatic disease did not contribute to the risk of IHCA. Similarly, the presence of cardiovascular risk factors was not associated with higher incidence of IHCA in cancer patients. Patients getting treatment with chemotherapy, immunotherapy and/or radiation therapy within six months preceding the admission were also found to have lower incidence of IHCA. 


\section{Additional Information \\ Disclosures}

Human subjects: Consent was obtained by all participants in this study. Institutional Review Board of Monmouth Medical Center issued approval 00003104. "Dr Louis Zinterhofer, Chairman, IRB, performed an expedited review on this study on 1/15/2018. He determined this protocol is exempt from further IRB continuing review". Animal subjects: All authors have confirmed that this study did not involve animal subjects or tissue. Conflicts of interest: In compliance with the ICMJE uniform disclosure form, all authors declare the following: Payment/services info: All authors have declared that no financial support was received from any organization for the submitted work. Financial relationships: All authors have declared that they have no financial relationships at present or within the previous three years with any organizations that might have an interest in the submitted work. Other relationships: All authors have declared that there are no other relationships or activities that could appear to have influenced the submitted work.

\section{References}

1. National vital statistic report-deaths: leading causes for 2015. (2017). Accessed: May 2018: https://www.cdc.gov/nchs/products/nvsr.htm.

2. Heymach J, Krilov L, Alberg A, et al.: Clinical cancer advances 2018: annual report on progress against cancer from the American Society of Clinical Oncology. J Clin Oncol. 2018, 36:1020-1044. 10.1200/JCO.2017.77.0446

3. Bilben B, Grandal L, Sovik S: National early warning score (NEWS) as an emergency department predictor of disease severity and 90-day survival in the acutely dyspneic patient: a prospective observational study. Scand J Trauma Resusc Emerg Med. 2016, 24:80. 10.1186/s13049-016-0273-9

4. Churpek M, Yuen T, Winslow C, et al.: Multicenter development and validation of a risk stratification tool for ward patients. Am J Respir Crit Care Med. 2014, 190:649-655.

5. Bartkowiak B, Snyder A, Benjamin A, et al.: Validating the electronic cardiac arrest risk triage (eCART) Score for risk stratification of surgical inpatients in the postoperative setting: retrospective cohort study. Ann Surg. 2018, [Epub ahead of print]:10.1097/SLA.0000000000002665

6. Bruckel J, Wong SL, Chan PS, Bradley SM, Nallamothu BK: Patterns of resuscitation care and survival after in-hospital cardiac arrest in patients with advanced cancer. J Oncol Pract. 2017, 13:e821-e830. 10.1200/JOP.2016.020404

7. Deo R, Sotoodehnia N, Katz R, et al.: Cystatin C and sudden cardiac death risk in the elderly . Circ Cardiovasc Qual Outcomes. 2010, 3:159-164. 10.1161/CIRCOUTCOMES.109.875369

8. Cerasola G, Nardi E, Mulè G, et al.: Left ventricular mass in hypertensive patients with mild-to-moderate reduction of renal function. Nephrology. 2010, 15:203-210. 10.1111/j.1440-1797.2009.01178.x

9. Levin A, Thompson CR, Ethier J, et al.: Left ventricular mass index increase in early renal disease: impact of decline in hemoglobin. Am J Kidney Dis. 1999, 34:125-134.

10. Paoletti E, Bellino D, Cassottana P, Rolla D, Cannella G: Left ventricular hypertrophy in nondiabetic predialysis CKD. Am J Kidney Dis. 2005, 46:320-327. 10.1053/j.ajkd.2005.04.031

11. Whitman IR, Feldman HI, Deo R: CKD and sudden cardiac death: epidemiology, mechanisms, and therapeutic approaches. J Am Soc Nephrol. 2012, 23:1929-1939. 10.1681/ASN.2012010037

12. Mark PB, Johnston N, Groenning BA, et al.: Redefinition of uremic cardiomyopathy by contrast-enhanced cardiac magnetic resonance imaging. Kidney Int. 2006, 69:1839-1845. 10.1038/sj.ki.5000249

13. Briet M, Collin C, Karras A, et al.: Arterial remodeling associates with CKD progression. J Am Soc Nephrol. 2011, 22:967-974. 10.1681/ASN.2010080863

14. Pai AS, Giachelli CM: Matrix remodeling in vascular calcification associated with chronic kidney disease . J Am Soc Nephrol. 2010, 21:1637-1640. 10.1681/ASN.2010040349

15. Haider AW, Larson MG, Benjamin EJ, Levy D: Increased left ventricular mass and hypertrophy are associated with increased risk for sudden death. J Am Coll Cardiol. 1998, 32:1454-1459.

16. Sandroni C, Dell'anna A, Tujjar O, Geri G, Cariou A, Taccone FS: Acute kidney injury after cardiac arrest: a systematic review and meta-analysis of clinical studies. Minerva Anestesiol. 2016, 82:989-999.

17. Lam AQ, Humphreys BD: Onco-nephrology: AKI in the cancer patient. Clin J Am Soc Nephrol. 2012, 7:16921700. 10.2215/CJN.03140312

18. Samuels J, Ng CS, Nates J, et al.: Small increases in serum creatinine are associated with prolonged ICU stay and increased hospital mortality in critically ill patients with cancer. Support Care Cancer. 2011, 19:15271532. 10.1007/s00520-010-0978-7

19. Møller S, Bernardi M: Interactions of the heart and the liver . Eur Heart J. 2013, 34:2804-2811. 10.1093/eurheartj/eht246

20. Abdelghani SA, Rosenthal TM, Morin DP: Surface electrocardiogram predictors of sudden cardiac arrest. Ochsner J. 2016, 16:280-289.

21. Morgan RW, Fitzgerald JC, Weiss SL, Nadkarni VM, Sutton RM, Berg RA: Sepsis-associated in-hospital cardiac arrest: epidemiology, pathophysiology, and potential therapies. J Crit Care. 2017, 40:128-135.

22. Dagher GA, El Khuri C, Chehadeh AA, et al.: Are patients with cancer with sepsis and bacteraemia at a higher risk of mortality? A retrospective chart review of patients presenting to a tertiary care centre in Lebanon. BMJ Open. 2017, 7:013502. 10.1136/bmjopen-2016-013502

23. Bleicher RJ, Ruth K, Sigurdson ER: Time to surgery and breast cancer survival in the United States . JAMA Oncol. 2016, 2:330-339. 10.1001/jamaoncol.2015.4508

24. Sara JD, Eleid MF, Gulati R, Holmes DR Jr: Sudden cardiac death from the perspective of coronary artery disease. Mayo Clin Proc. 2014, 89:1685-1698. 10.1016/j.mayocp.2014.08.022

25. Yannopoulos D, Bartos JA, Raveendran G, et al.: Coronary artery disease in patients with out-of-hospital refractory ventricular fibrillation cardiac arrest. J Am Coll Cardiol. 2017, 70:1109-1117. 


\section{Cureus}

10.1016/j.jacc.2017.06.059 